\title{
Stochastic optimisation-based valuation of smart grid options under firm DG contracts
}

\author{
Spyros Giannelos, Student Member IEEE, Ioannis Konstantelos, Member IEEE, and Goran Strbac, Member IEEE \\ Department of Electrical and Electronic Engineering \\ Imperial College London \\ London, United Kingdom
}

\begin{abstract}
Under the current EU legislation, Distribution Network Operators (DNOs) are expected to provide firm connections to new DG, whose penetration is set to increase worldwide creating the need for significant investments to enhance network capacity. However, the uncertainty around the magnitude, location and timing of future DG capacity renders planners unable to accurately determine in advance where network violations may occur. Hence, conventional network reinforcements run the risk of asset stranding, leading to increased integration costs. A novel stochastic planning model is proposed that includes generalized formulations for investment in conventional and smart grid assets such as Demand-Side Response (DSR), Coordinated Voltage Control (CVC) and Soft Open Point (SOP) allowing the quantification of their option value. We also show that deterministic planning approaches may underestimate or completely ignore smart technologies.
\end{abstract}

Index Terms - Firm DG contracts, Option Value of smart technologies, Planning under uncertainty, Real Options

\section{NOMENCLATURE}

\section{Sets and indices}

$\Omega_{E} \quad$ Set of epochs, indexed $e$

$\Omega_{G} \quad$ Set of generation units, indexed $g$

$\Omega_{L} \quad$ Set of distribution lines, indexed $l$

$\Omega_{C} \quad$ Set of normally-open points, indexed $c$

$\Omega_{M} \quad$ Set of scenario-tree nodes, indexed $m$

$\Omega_{N} \quad$ Set of system buses, indexed $n$

$\Omega_{T} \quad$ Set of demand periods, indexed $t$

$\varepsilon_{m} \quad$ Epoch to which scenario-tree node $m$ belongs

$\Phi_{k}(m)$ Time-ordered set of all parent nodes of scenario-tree node $m$, from the first epoch up to epoch $\varepsilon_{m}-k$

\section{Input Parameters}

$\gamma_{x} \quad$ Investment cost (£/year) for line reconductoring $(x=B), \operatorname{CVC}(x=C), \operatorname{DSR}(x=D), \operatorname{SOP}(x=S)$

$\eta_{f} \quad$ SOP efficiency factor

$\pi_{m} \quad$ Probability of scenario-tree node $m$ occurring

$\Psi_{n, t} \quad$ Tangent of the load angle at bus $n$ at period $t$

$\zeta_{t, g} \quad$ Output of $g \in \Omega_{D G}$ at $t$ expressed as $\%$ of its installed capacity. For $g \in \Omega_{T H}, \zeta_{t, g}=1, \forall t$

$b_{l}^{o} \quad$ Line susceptance before reinforcement (pu)
$b_{l}^{\mathrm{N}} \quad$ Line susceptance after reinforcement (pu)

$g_{l}^{\text {o }} \quad$ Line conductance before reinforcement $(\mathrm{pu})$

$g_{l}^{N} \quad$ Line conductance after reinforcement $(\mathrm{pu})$

$F_{l} \quad$ Existing capacity of line $l(\mathrm{pu})$

$F_{\max }$ Extra capacity, obtained from reconductoring, relative to the existing capacity $(\mathrm{pu})$

$\bar{D}_{t, n} \quad$ Max load that can be shifted to $t$ at bus $n(\mathrm{pu})$

$f_{t, n} \quad \%$ of initial load at $n$ for shifting to a period $\tau \neq t$

$I_{n, g} \quad$ Signifies if generator $g$ is connected to bus $n$

$d_{t, n} \quad$ Real power demand at bus $n$, period $t(\mathrm{pu})$

$k_{X} \quad$ Build time (epochs) for reconductoring $(x=L)$, for CVC $(x=C)$, DSR $(x=D)$, SOP $(x=S)$

$n_{c}^{x} \quad$ Terminals $(x=a, b)$ of SOP installed at $\mathrm{c}$

$P_{m, g}^{\max } \quad$ Max real power stable generation of $g(\mathrm{pu})$

$Q_{m, g}^{\max } \quad$ Max reactive power stable generation of $g(\mathrm{pu})$

$r_{\varepsilon_{m}}^{I}$

$S_{C}^{\max }$

$u_{l}$

Cumulative discount factor for investment cost

Capacity of SOP installed at $c$ (pu)

Sending bus of line $l$

Receiving bus of line $l$

$v_{l}$

$V_{\min }$

Fixed voltage value at the substation $(\mathrm{pu})$

Minimum voltage statutory limit (pu)

Maximum voltage statutory limit (pu)

Minimum voltage attainable by CVC (pu)

Maximum voltage attainable by $\mathrm{CVC}(\mathrm{pu})$

\section{Decision Variables}

$\theta_{m, t, n} \quad$ Voltage angle corresponding to bus $n$ (rad)

$\xi_{m, t, n} \quad$ Re-scheduled flexible demand at bus $n$ (pu)

$B_{m, l} \quad$ Binary variable for deciding to reconductor $l$

$\tilde{B}_{m, l} \quad$ State variable of reconductoring line $l$

$C_{m} \quad$ Binary variable for deciding to invest in CVC

$\tilde{C}_{m} \quad$ State variable of CVC investment

$D_{m, n} \quad$ Binary variable for deciding to invest DSR at $n$

$\widetilde{D}_{m, n} \quad$ State variable of DSR investment at bus $n$

$\tilde{F}_{m, l} \quad$ State variable representing the extra capacity due to reconductoring of line $l(\mathrm{pu})$

$H_{m, t, c} \quad$ Power drawn by SOP at terminal $n_{c}^{a}(\mathrm{pu})$

$R_{m, t, c} \quad$ Power drawn by SOP at terminal $n_{c}^{b}(\mathrm{pu})$ 


$\begin{array}{ll}P_{m, t, g} & \text { Real power output of unit } g(\mathrm{pu}) \\ P_{m, t, l}^{s} & \text { Real power flow at sending bus of line } l(\mathrm{pu}) \\ P_{m, t, l}^{r} & \text { Real power flow at receiving bus of line } l(\mathrm{pu}) \\ Q_{m, t, g} & \text { Reactive power output of unit } g(\mathrm{pu}) \\ Q_{m, t, l}^{s} & \text { Reactive power flow at sending bus of } l(\mathrm{pu}) \\ Q_{m, t, l}^{r} & \text { Reactive power flow at receiving bus of } l(\mathrm{pu}) \\ S_{m, c} & \text { Binary variable for deciding to invest in SOP } \\ \tilde{S}_{m, c} & \text { State variable of SOP investment } \\ V_{m, t, n} & \text { Voltage magnitude at bus } n(\mathrm{pu}) \\ V_{m, t}^{C} & \text { Substation voltage as regulated by CVC }(\mathrm{pu}) \\ V_{m, t}^{n o C} & \text { Substation voltage in absence of CVC }(\mathrm{pu})\end{array}$

\section{INTRODUCTION}

Distribution networks are facing multiple challenges due to the increasing penetration of renewables Distributed Generation (DG), which are currently expected to be integrated in the form of firm connections [1]. One such challenge relates to the development of the voltage rise effect which involves voltage magnitudes rising above statutory limits at times of low demand and high DG output. To face this challenge, one possible solution is for the Distribution Network Operators (DNOs) to resort to reinforcements of particular weak areas of the networks. However, since there is a lot of uncertainty around the location of future DG connections, given that they realize without prior coordination with the network planners, the DNOs cannot accurately determine in advance the location of occurrence of network violations and, therefore, some of the investment decisions may turn out stranded or underutilized.

Smart technologies may be viewed as an alternative to conventional network reinforcements [2] for tackling the voltage rise effect. In addition, due to their inherent flexibility they can allow risk management by reducing the potential for stranded conventional assets as they can be included in strategies that allow investments on a conditional basis. From this perspective, smart technologies can be viewed as real options [3]. Examples of such technologies include the Coordinated Voltage Control (CVC) [4],[9] Soft-Open Points (SOP) [5] and Demand-side Response (DSR) [6][7]. It is shown in this paper that since deterministic planning approaches cannot capture the managerial flexibility, these smart technologies are largely ignored; such an observation shows the importance of departing from current deterministic planning approaches in order to reduce spending on conventional assets and increase the integration of smart technologies.

According to the principles of connection of DG units to the network [8] curtailing the output of DG units, during network constraint situations, is possible for those DG units with non-firm rights. Although this practice is considered to be an active network management method [9], current EU legislation encourages the 'traditional' firm DG connections in order to fulfil the obligations from the EU 20-20-20 target [10]. The contributions of this paper are as follows:
- Presentation of mathematical formulations for investing and operating SOP, DSR and CVC assets in distribution networks.

- Development of a methodology that allows the quantification of the option value of smart technologies in distribution networks with firm DG connections.

- Demonstration of the inadequacy of deterministic planning approaches to favour smart technologies (SOP, CVC, DSR) in place of conventional ones.

The remaining sections of this paper are organized as follows: Section III. reviews the existing literature and gives insights regarding methodologies on the quantification of real option value. Section IV. presents the mathematical formulation and Section V. shows the application of the proposed planning framework to an $11 \mathrm{kV}$ distribution network. Section VI. offers conclusions and recommendations for future work.

\section{REAL OPTION VALUATION FRAMEWORKS}

Real Options Analysis has been utilized as a tool for the evaluation of flexibility in various decision-making problems with the option value of making a certain decision being traditionally defined as the benefit that the decision maker accrues from making the decision under uncertainty [11]. Uncertainty and irreversibility have long been recognized as principal factors driving the option value up [12]. Thus, distribution network planning undoubtedly incorporates option value, with various techniques being applied for its quantification. In [13], the option value of investment strategies is obtained through the Least Squares Monte Carlo approach, an approach that is also used in [14][11]. In [15], the use of the Binomial Lattice Model allows the computation of the real option to expand in generation planning, while in [16] the Black - Scholes option pricing formula is applied to the valuation of a power generation construction project.

The drawback of using valuation frameworks as the aforementioned is that they can evaluate only a small number of candidate investment strategies, which are defined in advance in terms of the technologies that they consist of and in terms of the order at which these technologies are invested. In order to compare and evaluate the numerous combinations of asset types, candidate locations and possible investment timings, the use of multistage stochastic optimization is imperative.

\section{MATHEMATICAL FORMULATION}

The model is formulated as a stochastic mixed integer nonlinear problem using a multi-stage scenario tree of $\left|\Omega_{\mathrm{M}}\right|$ nodes over $\left|\Omega_{\mathrm{E}}\right|$ epochs to represent uncertainty around DG penetration.

$z=\min _{B, C, D, S}\left\{\sum_{m \in \Omega_{M}} \pi_{m} r_{\varepsilon_{m}}^{I} \omega_{m}^{I}\right\}$
$\omega_{m}^{I}=C_{m} \gamma_{C}+\sum_{l \in \Omega_{L}} B_{m, l} \gamma_{B}+\sum_{n \in \Omega_{N}} D_{m, n} \gamma_{D}+\sum_{c \in \Omega_{C}} S_{m, c} \gamma_{S}$ 


$$
\begin{aligned}
& \tilde{B}_{m, l}=\sum_{\varphi \in \Phi_{k_{L}}(m)} B_{\varphi, l} \\
& \tilde{F}_{m, l}=\sum_{\varphi \in \Phi_{k_{L}}(m)} B_{\varphi, l} F_{\max } \\
& \tilde{C}_{m}=\sum_{\varphi \in \Phi_{k_{C}}(m)} C_{\varphi} \\
& \widetilde{D}_{m, n}=\sum_{\varphi \in \Phi_{k_{D}}(m)} D_{\varphi, n} \\
& \tilde{S}_{m, c}=\sum_{\varphi \in \Phi_{k_{s}}(m)} S_{\varphi, c} \\
& P_{m, t, 1} \leq P_{m, 1}^{\max } \\
& Q_{m, t, 1} \leq Q_{m, 1}^{\max } \\
& P_{m, t, g}=P_{m, g}^{\max } \cdot \zeta_{t, g} \\
& Q_{m, t, g}=Q_{m, g}^{\max } \cdot \zeta_{t, g} \\
& P_{m, t, l}^{S}=\left(1-\tilde{B}_{m, l}\right)\left[V_{m, t, u_{l}}^{2} g_{l}^{o}-V_{m, t, u_{l}} V_{m, t, v_{l}} g_{l}^{o} .\right. \\
& \left.\cos \left(\theta_{m, t, u_{l}}-\theta_{m, t, v_{l}}\right)-V_{m, t, u_{l}} V_{m, t, v_{l}} b_{l}^{o} \sin \left(\theta_{m, t, u_{l}}-\theta_{m, t, v_{l}}\right)\right] \\
& +\tilde{B}_{m, l}\left[V_{m, t, u_{l}}^{2} g_{l}^{N}-V_{m, t, u_{l}} V_{m, t, v_{l}} g_{l}^{N} \cos \left(\theta_{m, t, u_{l}}-\theta_{m, t, v_{l}}\right)\right. \\
& \left.-V_{m, t, u_{l}} V_{m, t, v_{l}} b_{l}^{N} \cdot \sin \left(\theta_{m, t, u_{l}}-\theta_{m, t, v_{l}}\right)\right] \\
& P_{m, t, l}^{r}=\left(1-\tilde{B}_{m, l}\right)\left[V_{m, t, v_{l}}^{2} g_{l}^{o}-V_{m, t, v_{l}} V_{m, t, u_{l}} g_{l}^{o} .\right. \\
& \left.\cos \left(\theta_{m, t, v_{l}}-\theta_{m, t, u_{l}}\right)-V_{m, t, u_{l}} V_{m, t, v_{l}} b_{l}^{o} \sin \left(\theta_{m, t, v_{l}}-\theta_{m, t, u_{l}}\right)\right] \\
& +\tilde{B}_{m, l}\left[V_{m, t, v_{l}}^{2} g_{l}^{N}-V_{m, t, u_{l}} V_{m, t, v_{l}} g_{l}^{N} \cos \left(\theta_{m, t, v_{l}}-\theta_{m, t, u_{l}}\right)\right. \\
& \left.-V_{m, t, u_{l}} V_{m, t, v_{l}} b_{l}^{N} \cdot \sin \left(\theta_{m, t, v_{l}}-\theta_{m, t, u_{l}}\right)\right] \\
& Q_{m, t, l}^{s}=\left(1-\tilde{B}_{m, l}\right)\left[-V_{m, t, u_{l}}^{2} b_{l}^{o}-V_{m, t, u_{l}} V_{m, t, v_{l}} g_{l}^{o}\right. \text {. } \\
& \left.\sin \left(\theta_{m, t, u_{l}}-\theta_{m, t, v_{l}}\right)+V_{m, t, u_{l}} V_{m, t, v_{l}} b_{l}^{o} \cos \left(\theta_{m, t, u_{l}}-\theta_{m, t, v_{l}}\right)\right] \\
& +\tilde{B}_{m, l}\left[-V_{m, t, u_{l}}^{2} b_{l}^{N}-V_{m, t, u_{l}} V_{m, t, v_{l}} g_{l}^{N} \sin \left(\theta_{m, t, u_{l}}-\theta_{m, t, v_{l}}\right)\right. \\
& \left.+V_{m, t, u_{l}} V_{m, t, v_{l}} b_{l}^{N} \cos \left(\theta_{m, t, u_{l}}-\theta_{m, t, v_{l}}\right)\right] \\
& +\tilde{B}_{m, l}\left[-V_{m, t, v_{l}}^{2} b_{l}^{N}-V_{m, t, u_{l}} V_{m, t, v_{l}} g_{l}^{N} \sin \left(\theta_{m, t, v_{l}}-\theta_{m, t, u_{l}}\right)\right. \\
& \left.+V_{m, t, u_{l}} V_{m, t, v_{l}} b_{l}^{N} \cos \left(\theta_{m, t, v_{l}}-\theta_{m, t, u_{l}}\right)\right] \quad \forall m, t, l \\
& \left(P_{m, t, l}^{s, r}\right)^{2}+\left(Q_{m, t, l}^{s, r}\right)^{2} \leq\left[F_{l}+\widetilde{F}_{m, l}\right]^{2} \\
& V_{m, t, 1}=V_{m, t}^{c v c}+V_{m, t}^{n o c} \\
& V_{\min }^{c v c} \cdot \tilde{C}_{m} \leq V_{m, t}^{c v c} \leq V_{\max }^{c v c} \cdot \tilde{C}_{m} \\
& V_{m, t}^{n o c}=V_{s e t} \cdot\left(1-\tilde{C}_{m}\right) \\
& -\widetilde{D}_{m, n} \cdot f_{t, n} \cdot d_{t, n} \leq \xi_{m, t, n} \leq \widetilde{D}_{m, n} \bar{D}_{t, n} \\
& \sum_{t \in \Omega_{T}} \xi_{m, t, n}=0 \\
& \forall m, t, n-\{1\}
\end{aligned}
$$

$\sum_{c \in\left\{\Omega_{C} \mid n=n_{c}^{b}\right\}}\left(R_{m, t, c}\right.$

$$
\left.-H_{m, t, c} \eta_{f}\right)
$$

$\forall m, t, n(25)$ $\sum_{g \in \Omega_{g}} Q_{m, t, g} I_{n, g}-\sum_{l \in\left\{\Omega_{L} \mid v_{l}=n\right\}} Q_{m, t, l}^{r}-\sum_{l \in\left\{\Omega_{L} \mid u_{l}=n\right\}} Q_{m, t, l}^{s}=$
$+\Psi_{n, t}\left(d_{t, n}+\xi_{m, t, n}\right)$

$\forall m, t, n(26)$

The planner's objective (1) involves the minimization of the expected investment cost (2) across all scenario-tree nodes and technologies. This stochastic problem formulation is known as node-variable (see [17] for more information) and has been shown to be computationally effective. The binary variables $B_{m, l}, D_{m, n}, S_{m, c}, C_{m}$ represent the decisions to invest in conventional reinforcement, DSR, SOP, and CVC respectively at node $m$ of the scenario tree, where $l, n, c$ characterize the line, bus and normally open point respectively. Each binary investment decision is multiplied by the corresponding annualized investment cost. Note that a different investment decision corresponds to each scenario tree node $m$, indicating the possibility for strategy differentiation between scenario realisations. The state variables that aggregate investment decisions that have been made are defined in (3)-(7). The primary transformer $(\mathrm{g}=1)$ thermal limits are stated in (8)-(9), while (10) and (11) determine the renewable DG output where intermittency of the resource is considered by $\zeta_{t, g}$. The polar form of the power flows is given in (12)-(15) using a disjunction to model the effect that reconductoring has on a line's characteristics $\mathrm{b}_{1}$ and $g_{l}$. Line thermal limitation is defined in (16) while (17) sets the limits on voltage magnitudes for all buses, except for the substation which is fixed to a pre-determined value $V_{\text {set }}(18)$. Constraints (19) and (20) model the CVC deployment, after which the substation voltage is no longer fixed, but can be controlled to take values in the domain $\left[\mathrm{V}_{\min }^{\mathrm{cvc}}, \mathrm{V}_{\max }^{\mathrm{cvc}}\right]$. In more detail, the voltage magnitude at the substation is defined in (18) as follows: when the CVC scheme has not been deployed $\left(\tilde{C}_{m}=0\right)$ a fixed voltage-target policy is assumed, where $V_{m, t, 1}=$ $V_{m, t}^{n o c}=V_{s e t}, V_{m, t}^{c v c}=0$ according to (18-20). If a CVC scheme has been deployed $\left(\tilde{C}_{m}=1\right)$, then the substation voltage target no longer follows a fixed voltage-target policy. Rather, it can be controlled optimally based on real-time information about system voltages; in this case it is $V_{m, t, 1}=V_{m, t}^{c v c}$, with $V_{\min }^{c v c} \leq V_{m, t}^{c v c} \leq V_{\max }^{c v c}$ according to (18)-(19).

The DSR operation is modeled in (21) and (22) where positive values of $\xi_{\mathrm{m}, \mathrm{t}, \mathrm{n}}$ reflect that flexible demand has been re-scheduled from another period to the current period $t$, whereas negative values signify that flexible demand has been shifted away from $\mathrm{t}$ to another period. In particular, in (21) the load that is disconnected at period $t$ from bus $n$ at node $m$ is zero if no DSR has been deployed at bus $n$ i.e. if $\widetilde{D}_{m, n}=0$. Otherwise, it can attain values within $f_{t, n} \cdot d_{t, n}$ and $\bar{D}_{t, n}$ i.e. between the total amount of flexible load available at time period $t$, and the total amount of load that can connect to bus $\mathrm{n}$ at period $\mathrm{t}$, respectively. Load shifting operation is performed so that all flexible load is 
eventually served within the period of a typical day as shown by (22). Also (23) - (24) refer to SOP, installed at normally-open point $c$. This SOP enables bi-directional transfer of active power between its two buses $n_{c}^{a}$ and $n_{c}^{b}$ with efficiency $\eta_{f}$. This transfer has to respect the SOP active power transfer limits according to constraints (23) - (24). According to these constraints, if the SOP has not been deployed at $\mathrm{c}$ then the corresponding controlled power flows must be zero i.e. $R_{m, t, c}=H_{m, t, c}=0$. Note that reactive power capability of SOPs has not been modelled in this paper. Finally, (25) - (26) define the power balance equations.

\section{CASE STUdY}

The case study displays the methodology of obtaining the option value of smart technologies such as DSR, CVC and SOP under uncertain renewable DG deployment and under the constraint that curtailment of DG output has to be avoided (firm DG connection). It is also shown that these technologies are ignored by deterministic planning frameworks, which favor risky conventional investment.

\section{A. Description}

The $11 \mathrm{kV}$ overhead distribution network used in the case study is shown in Figure 1., where initially all system demand is satisfied through imports from the main grid via bus 1 , which is the primary substation. The statutory voltage limits at all buses are assumed to be $\pm 0.1 \mathrm{pu}$. An uncertain amount of distributed PV generation (see Figure 3) will be connected over the planning horizon, which consists of four epochs each of 2-year duration. However, the planner does possess a probabilistic description of this uncertain evolution, as expressed in the scenario tree shown in Figure 3. This scenario tree consists of a total of six scenarios; there are six possible paths from the first 'root' node to a terminal 'leaf' node. For example, scenario 2 (S2) consists of the transition across the scenario tree nodes $1 \rightarrow 2 \rightarrow 4 \rightarrow 9$, representing PV deployment in buses 5,6 and 7. As can be seen, the uncertainty is not only around the magnitude of the DG penetration, but also around the location of these connections.

We assume that the tree shown in Figure 3 has been constructed following suitable consultation with developers and system experts. The logic followed in the scenario tree generation is as follows. In the first stage i.e. root node, there is no PV deployed in the system. However, in the subsequent stages there will be PV deployment in either feeder F-1 or F-2. The uncertainty of which one of these two feeders will be fitted with $\mathrm{PV}$ is resolved in transition to the second stage. In the case of a 1 $\rightarrow 2$ transition, buses 6 and 7 on F-1 are fitted with PV. Subsequent transitions in stages 3 and 4 will determine whether this will remain unchanged (as described by scenario 4) or additional PV capacity will be added. Note that the general philosophy is that PV is first built in the most distant buses and may eventually be deployed at buses closer to the substation.

As the voltage rise effect is the only driver for investment, the focus of the analysis is placed on the day that leads to the most severe voltage rise. Identifying a scheme that resolves this issue guarantees the ability for unconstrained operation at all other operating points. This is a typical approach taken to alleviate the computational load of planning studies without compromising solution integrity (e.g. see [9]). The day that leads to the most severe voltage rise is assumed to be a summer day with very high solar insolation (hence, output of the PV units) and very low demand levels; these conditions are appropriate for the creation of the voltage rise effect. Regarding the demand pattern, it is assumed to stay unchanged across the horizon (i.e. no load growth in subsequent epochs). Also, all buses have an identical electricity consumption profile and magnitude as shown in Figure 2 with the exception of the buses 8-11 on F-2 that have significantly lower electricity demand and their load is assumed to be zero. Furthermore, it has been assumed that the substation voltage setpoint is at $1.01 \mathrm{pu}$; this value is traditionally selected above 1 pu to prevent voltage drop at remote buses.

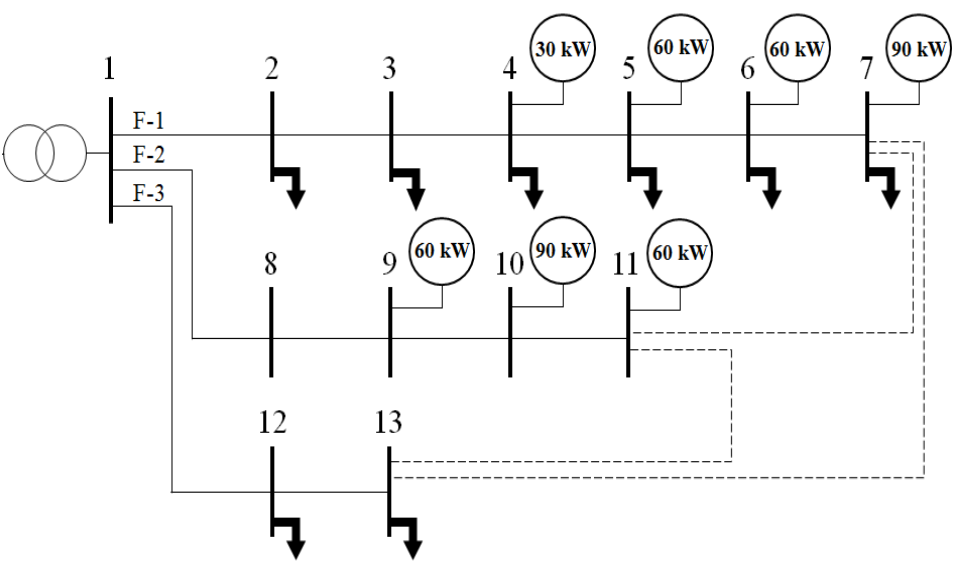

Figure 1. Schematic diagram of the $11 \mathrm{kV}$ distribution network, showing possible locations for the connection of the distributed PV units and their installed capacities. Dotted lines depict the Normally-Open Points (NOPs), which are candidate locations for SOPs. The F-1, F-2 and F-3 are the three feeders comprising the network.

Since the PV units reach their maximum output at midday (see Figure 2), the voltage rise effect is especially likely to occur around midday hours. In order to prevent its occurrence, the planner has a range of potential solutions shown in TABLE I.

The investment cost of the different technologies has been estimated according to relevant sources (see [18] and [19][19]), while the difference in build-time between the smart and conventional assets can be attributed to the fact that the latter involve greater network intervention in the form of necessary public works, which can be subject to lengthy permissioning processes.

The DSR technology allows the optimal intraday timeshifting of the flexible load, which amounts to $20 \%$ of the hourly load of each bus. It is modelled by the constraints (21), (22), (25) and (26) in the previous section. The CVC technology can measure the actual voltage values at all buses in the network, enabling the optimal regulation of the substation voltage by $\pm 5 \%$ of the nominal voltage setpoint (set at $1.01 \mathrm{pu}$ ). This technology is modelled by (18), (19), and (20) in the previous section. The SOP 
technology allows optimal control of active power flow through its two terminals; $95 \%$ efficiency and $50 \mathrm{~kW}$ capacity are used in this case study. It is modelled by (23), (24) and (25) in the previous section. The reconductoring of a distribution line involves the replacement of the existing conductor (reactance $\mathrm{X}=$ $0.075 \Omega / \mathrm{km}$, resistance $\mathrm{R}=0.250 \Omega / \mathrm{km})$ with a new one $(\mathrm{X}=$ $0.0725 \Omega / \mathrm{km}$ and $\mathrm{R}=0.0736 \Omega / \mathrm{km}$ ) as modelled by constraints (12)-(15). These characteristics are based on the specifications included in the E.ON's Network Design Manual v7.7, Dec. 2006 [19] and are in line with the technical characteristics of conductors available on the market. Note that the PV units do not perform reactive management due to unity power factor operation.

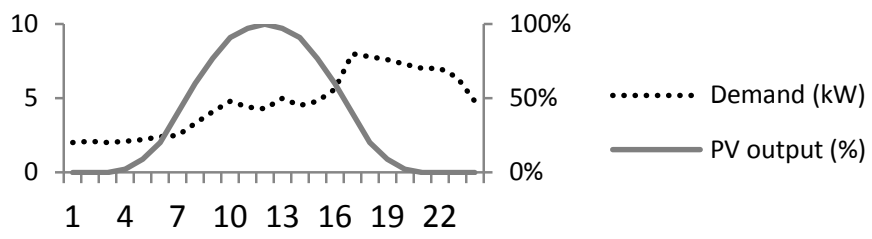

Figure 2. Generation pattern per PV unit (right axis) and demand profile per bus (left axis).

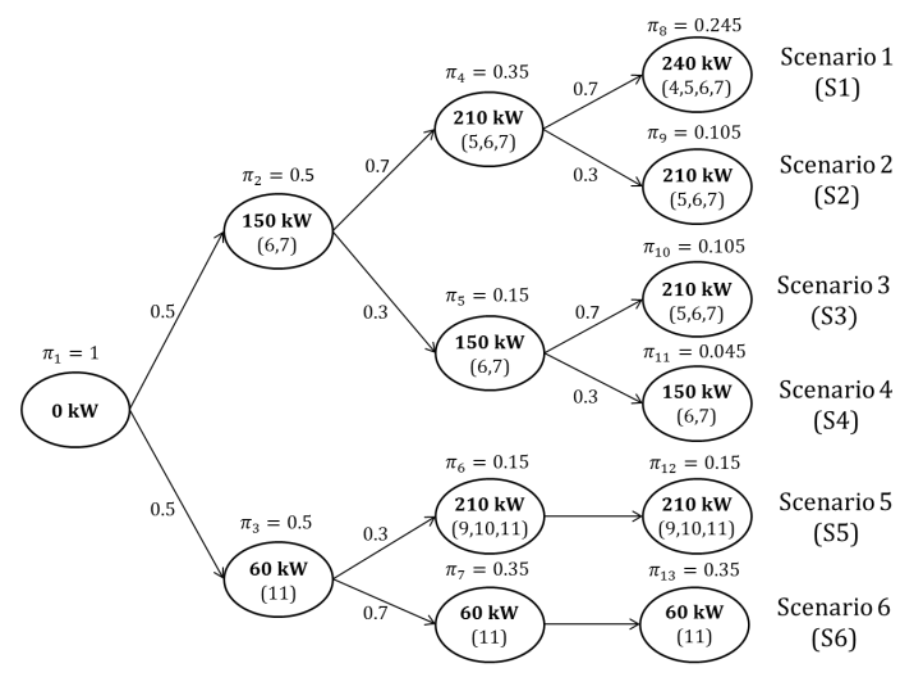

Figure 3. Scenario tree capturing the uncertainty of total PV capacity. Transition probabilities (above each arc) and event probabilities $\left(\pi_{m}\right.$, where $m$ is the number of the node) are shown. The aggregate PV capacity installed and the buses to which the PV units connect, are shown inside each node.

TABLE I. AVAILABLE TECHNOLOGIES FOR INVESTMENT
\begin{tabular}{|c|c|c|}
\hline Technology & Build Time (epochs) & Investment Cost (千) \\
\hline DSR & 0 & $14 \mathrm{k} /$ bus \\
\hline CVC & 0 & $180 \mathrm{k} /$ whole system \\
\hline SOP & 0 & $200 \mathrm{k} /$ NOP \\
\hline Reconductoring & 1 & $210 \mathrm{k} / \mathrm{km}$ \\
\hline
\end{tabular}

A number of deterministic and stochastic studies are performed using the model presented in Section IV. . All models were developed using FICO Xpress 7.8 and carried out on a Xeon $3.46 \mathrm{GHz}$ computer.

\section{B. Deterministic planning}

A deterministic planner traditionally obtains the investment schedule by solving separately each of the six scenarios ( 1 1 - S6) depicted in Figure 3 by applying the model described in Section IV and setting the $\pi_{\mathrm{m}}$ probabilities equal to 1 (no uncertainty exists, i.e. perfect information). Note that the planner can invest in all technologies shown in TABLE I. The resulting investment plans for each scenario are displayed in Figure 4. Note that no averaging over the scenario tree takes place (as is usually the case in a deterministic-equivalent approach); instead a separate study is carried out for each scenario. The [a-b] represents the decision to invest in reconductoring of the line that connects buses a and $b$, while $D(n)$ represents the decision to invest in DSR at bus $n$. Entries of the form $\operatorname{SOP}(\mathrm{a}-\mathrm{b})$ represent the decision to invest in a SOP at the normally-open point between buses $\mathrm{a}$ and $\mathrm{b}$, while CVC denotes installation of a CVC scheme in the substation.

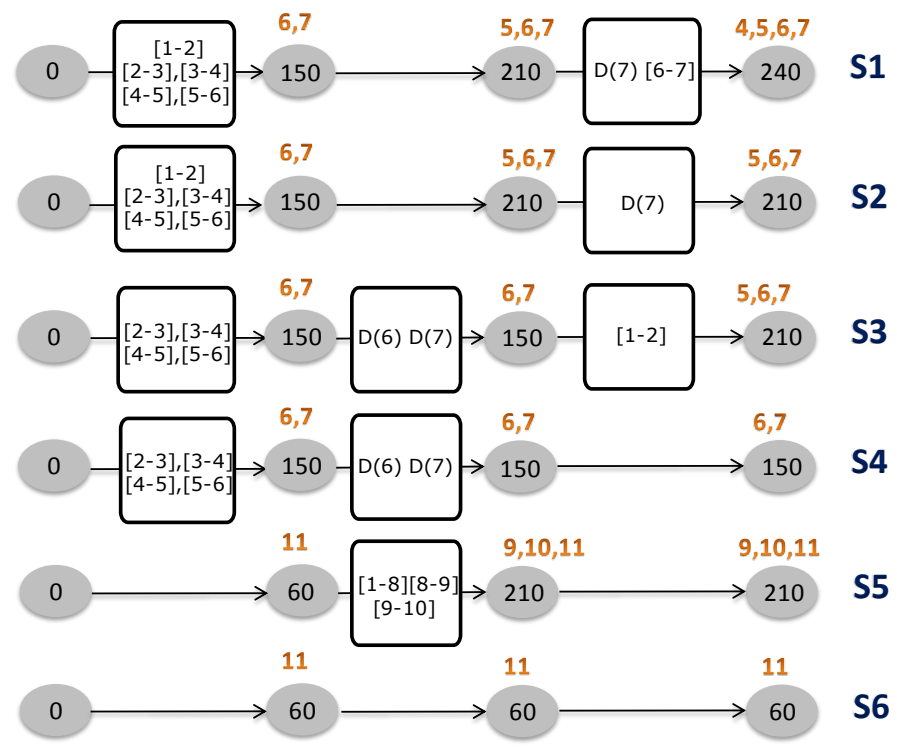

Figure 4. Investment plans for the deterministic planner. Each text box displays the investment decisions. The conventional investments (in the form [a-b]) become operational one epoch after the investment decision has been made, while the smart investments (in the form $D(n)$ ) become operational at the same epoch when the corresponding decisions are made.

The resulting investment plans are dominated by decisions to undertake conventional investments. Four out of the six scenarios involve conventional commitments made in the first epoch. This is particularly problematic because making the decision to invest in conventional reinforcements 'here and now', and assuming that there is no uncertainty, may lead a significant portion of these investments to become stranded assets. 
In addition, it is remarkable that SOP and CVC technologies are fully ignored - despite their availability to the planner- while DSR is deployed to a limited extent. This is unattractive because of the desire to make the grid smarter, something that can only be achieved through fewer conventional investments and more smart ones. However, given that initially the cost of smart technologies may be particularly high and that the planner cannot capture their option value (because no opportunity for conditional investments exists), they are not considered attractive.

\section{Stochastic planning}

As part of the stochastic planning two studies are carried out. In the first, line reconductoring is the only available investment technology. In the second study the planner can consider both the conventional and the smart technologies. The resulting investment strategies are shown in Figure 5 and Figure 6 respectively. It can be observed that the latter includes far fewer conventional investment decisions with no first-stage decisions because the presence of smart technologies enables the planner to commit only after the locational uncertainty of future PV development is resolved (e.g. separate strategies for the node $1 \rightarrow$ node 2 and node $1 \rightarrow$ node 3 branches), reducing the scope for asset stranding.

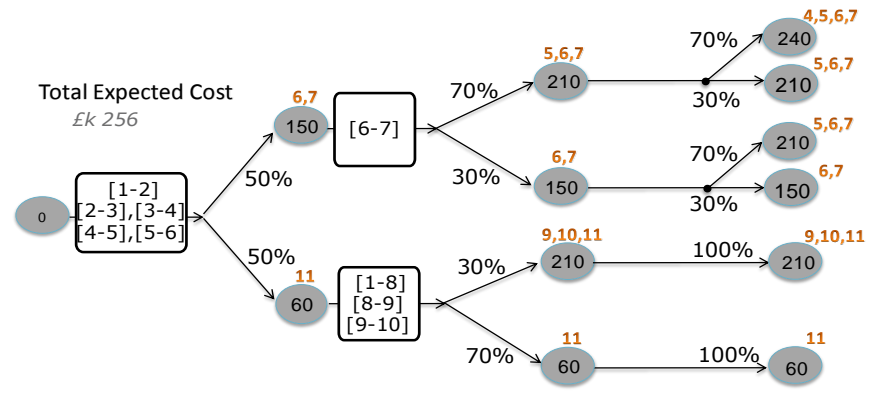

Figure 5. Investment strategy for the stochastic planner when only the conventional technology is available for investment.

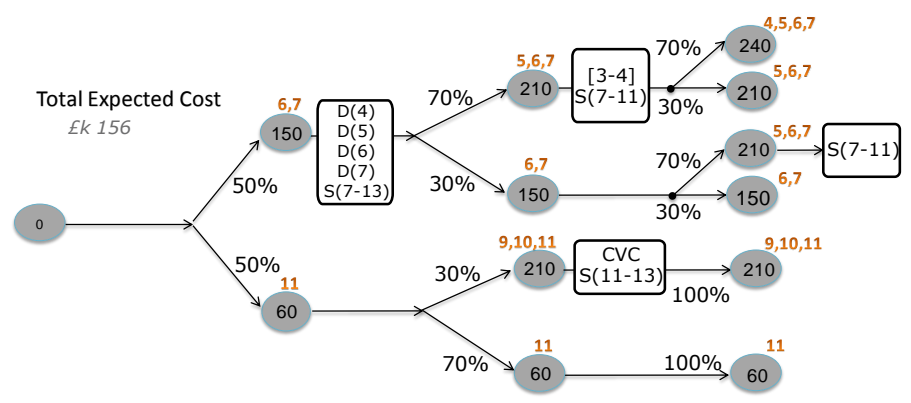

Figure 6. Investment strategy for the stochastic planner when conventional and smart technologies(CVC, SOP, DSR) are available for investment.

Figure 7 illustrates the operation of DSR in bus 7; flexible load of bus 7 is shifted from periods of small (or zero) PV generation to periods of higher PV generation, thereby reducing net power injections in the network. Figure 8 illustrates the management of voltage profiles during operation of node 6 , enabled by the installed CVC scheme. As shown, the voltage magnitude at bus 11 is kept within limits by reducing the substation voltage during hours of high PV output.

The basic operating principle of SOP technology for tackling the voltage rise effect is depicted in Figure 9, where the voltage magnitude at bus 7 is kept within limits as follows: at times of high PV generation, the SOP draws power from bus 7 and releases it to bus 13 . This increases the total demand at all buses of F-1 while simultaneously reducing that of F-3.

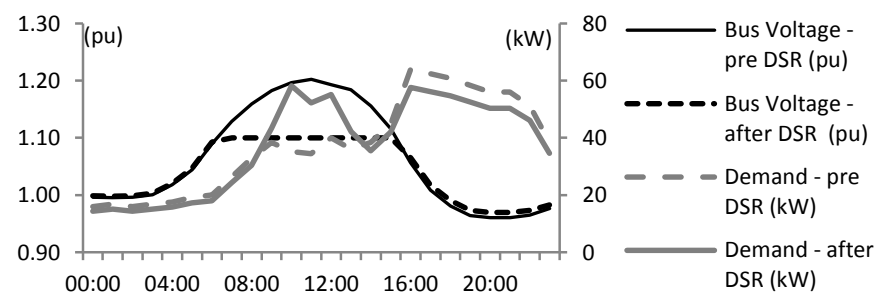

Figure 7. Impact of DSR operation of bus 7 on the load pattern (node 2)

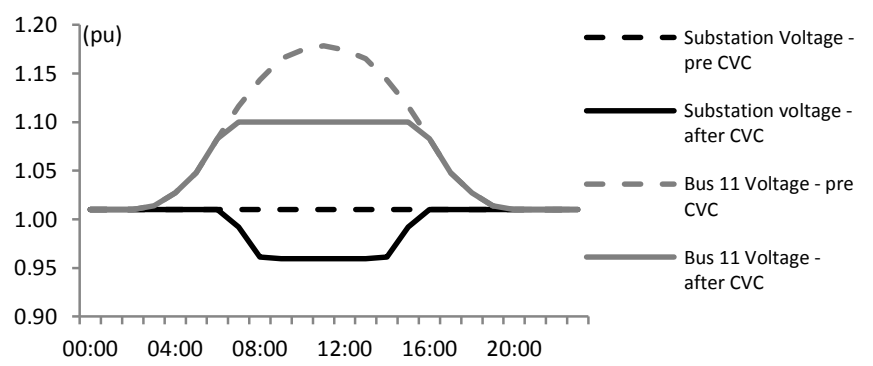

Figure 8. Impact of CVC operation on the voltage profile of bus 11 (node 6)

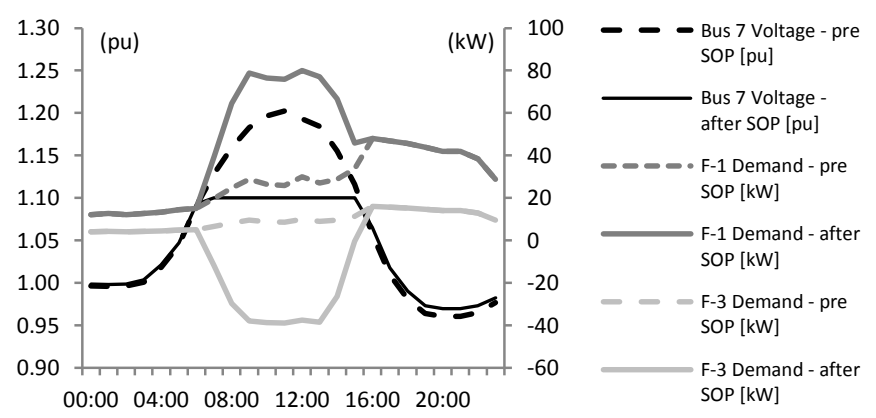

Figure 9. Impact of SOP operation on voltage profile of bus 7 and on the load profile of feeder-1 \& feeder-3, referring to scenario-tree node 2

By comparing the total expected cost of the strategies shown in Figure 5 and Figure 6, we can quantify the option value of the portfolio of all smart technologies being $£ 256 \mathrm{k}-£ 156 \mathrm{k}=£ 100 \mathrm{k}$ and represents the net benefit accrued from the ability to invest in smart assets. Three further stochastic studies are carried out, each considering the ability to invest in conventional assets and one of the three smart technologies. This way, we obtain the option value of DSR, CVC and SOP to be equal to $£ 24 \mathrm{k}, £ 33 \mathrm{k}$ and $£ 68 \mathrm{k}$. The 
sum of option values of individual technologies is greater than the option value of the combined portfolio, as the latter accounts for complementary interactions between individual assets.

In the proposed methodology the option being valued is the ability to invest in smart technologies. The value of this option is calculated to be $£ 100 \mathrm{k}$ for the particular network under study. This option value is defined as the probability-weighted sum of capital savings made possible by being able to invest in a particular technology.

Finally, all models were developed in FICO Xpress 7.8. For the solution of the Mixed-Integer Non-Linear Programming (MINLP) that arises, the "mmxnlp" module of the Xpress-NLP engine has been used. This solution strategy involves a combination of Sequential Linear Programing to approximate non-linear elements and a traditional Branch and Bound technique to identify optimal value of binary variables. All studies were carried out on a Xeon $3.46 \mathrm{GHz}$ computer. In general, the computational time depends on whether we carry out a deterministic or stochastic study and whether we include smart technologies or not. In particular, the six deterministic case studies took, on average, thirty minutes each. The stochastic planning case study took one hour when considering only conventional technologies. The computational burden is more pronounced in the stochastic study that considers both conventional and smart technologies; convergence was achieved in 22 hours.

\section{CONCLUSION AND FUTURE WORK}

This paper proposes a stochastic model for identifying the optimal investment strategy that will eradicate the voltage rise effect caused by increased DG penetration. In addition, the inadequacy of deterministic approaches is shown to incorporate smart technologies in their investment plans and their preference for immediate investments in conventional assets. Note that Heuristic rules based on deterministic decisions, such as identifying common elements across scenarios, also underperform; this fact that has been demonstrated in the context of congestion-driven transmission investment in [20].

Future work focuses on the application of decomposition techniques for achieving more efficient solution times in the same problem. Another future goal involves the investigation of riskaverse decision criteria for the detailed modelling of a planner's attitude towards the risk of stranded assets.

\section{REFERENCES}

[1] Eurelectric, Active Distribution System Management, Feb. 2013.

[2] O. Brewin, S.C.E. Jupe, M. G. Bartlett, K.T. Jackson and C. Hanmer, "New technologies for low voltage distribution networks", ISGT Europe 2011, Manchester, 2011.

[3] Ofgem, Real Options and Investment Decision Making, Mar. 2012.

[4] Kulmala, A.; Repo, S.; Järventausta, P., "Coordinated Voltage Control in Distribution Networks Including Several Distributed Energy Resources," in Smart Grid, IEEE Transactions on , vol.5, no.4, pp.2010-2020, July 2014
[5] Giannelos, S.; Konstantelos, I.; Strbac, G., "Option value of Soft Open Points in distribution networks," in PowerTech, 2015 IEEE Eindhoven, vol., no., pp.1-6, June 29 2015-July 22015

[6] Rabiee, A.; Soroudi, A.; Mohammadi-Ivatloo, B.; Parniani, M., "Corrective Voltage Control Scheme Considering Demand Response and Stochastic Wind Power," in Power Systems, IEEE Transactions on , vol.29, no.6, pp.2965-2973, Nov. 2014

[7] G. Strbac, "Demand side management: Benefits and challenges", Energy Policy, vol. 36, no. 12, pp. 4419-4426, 2008.

[8] Dolan, M.J.; Davidson, E.M.; Kockar, I.; Ault, Graham W.; McArthur, S.D.J., "Distribution Power Flow Management Utilizing an Online Optimal Power Flow Technique," in Power Systems, IEEE Transactions on , vol.27, no.2, pp.790-799, May 2012

[9] S.N. Liew and G. Strbac, "Maximizing Penetration of Wind Generation in Existing Distribution Networks", IEE Proceedings on Generation, Transmission and Distribution, vol. 149, no. 3, 2002.

[10] European Commission, Combating Climate Change: The EU Leads the Way. [Online]. Available: http://ec.europa.eu/publications/ booklets/move/70/index_en.htm.

[11] B.A. Weisbrod, "Collective-consumption services of individualconsumption goods", The Quarterly Journal of Economics, vol. 78, no. 3, pp. 471-477, 1964.

[12] A. Dixit and R. Pindyck, "Investment Under Uncertainty". Princeton, NJ: Princeton Univ. Press, 1994, pp. 93-125.

[13] E. Buzarquis, G. A. Blanco, F. Olsina, and F. Garces, "Valuing investments in distribution networks with DG under uncertainty," IEEE PES T\&D Conference and Exposition, Sao Paulo, 2010.

[14] G. Blanco, F. Olsina, F. Garces, and C. Rehtanz, "Real option valuation of FACTS investments based on the least square Monte Carlo Method," IEEE Trans. Power Syst., vol. 26, no. 3, pp. 1389-1398, Aug. 2011.

[15] Min, K.J.; Chung-Hsiao Wang, "Generation planning for inter-related generation units: a real options approach," in Power Engineering Society Summer Meeting, 2000. IEEE , vol.4, no., pp.2261-2265 vol. 4, 2000

[16] Yunna, Wu; Li Cong; Tang Qifen, "Application of real options to investment on power construction," in Logistics Systems and Intelligent Management, 2010 International Conference on , vol.1, no., pp.595-598, 9-10 Jan. 2010

[17] A.J. Conejo, M. Carrion, J.M. Morales, Decision Making under Uncertainty in Electricity Markets, New York: Springer, 2010.

[18] Frontier Economics, A framework for the evaluation of smart grids: A report prepared for Ofgem, Mar. 2012.

[19] E.ON, Network Design Manual v7.7, Dec. 2006.

[20] I. Konstantelos and G. Strbac, "Valuation of Flexible Investment Options under Uncertainty", IEEE Transactions on Power Systems - Special Section: Power System Planning and Operation towards a Low-Carbon Economy, 2014. 